October 26, 2018

\title{
LINEAR MATRIX INEQUALITY REPRESENTATION OF SETS
}

\author{
J. William Helton and Victor Vinnikov \\ Mathematics Dept. UCSD \\ Ben Gurion Univ. of the Negev \\ La Jolla Cal \\ 92093, USA \\ Beer Sheva, 84105 \\ Israel \\ helton@math.edu \\ vinnikov@math.bgu.ac.il
}

\begin{abstract}
This article concerns the question: which subsets of $\mathbb{R}^{m}$ can be represented with Linear Matrix Inequalities, LMIs? This gives some perspective on the scope and limitations of one of the most powerful techniques commonly used in control theory. Also before having much hope of representing engineering problems as LMIs by automatic methods one needs a good idea of which problems can and cannot be represented by LMIs. Little is currently known about such problems. In this article we give a necessary condition, we call "rigid convexity", which must hold for a set $\mathcal{C} \in \mathbb{R}^{m}$ in order for $\mathcal{C}$ to have an LMI representation. Rigid convexity is proved to be necessary and sufficient when $m=2$. This settles a question formally stated by Pablo Parrilo and Berndt Sturmfels in [PSprep].
\end{abstract}




\section{The Problem of Representing Sets with LMI's}

Given $\mathcal{C}$ a closed convex set in $\mathbb{R}^{m}$ bounded by algebraic hypersurfaces

$$
S_{p_{j}}:=\left\{x \in \mathbb{R}^{m}: p_{j}(x)=0\right\},
$$

with all polynomials $p_{j}(x)>0$ for $x$ in the interior of $\mathcal{C}$. Which $\mathcal{C}$ can be represented in terms of some linear pencil $L=\left\{L_{0}, L_{1}, L_{2}, \cdots, L_{m}\right\}$ as

$$
\mathcal{C}^{L}:=\left\{x=\left(x_{1}, x_{2}, \cdots x_{m}\right): L_{0}+L_{1} x_{1}+\cdots+L_{m} x_{m} \quad \text { is PosSemiDef }\right\} ?
$$

We shall require by our use of the term linear pencil that $L_{0}, L_{1}, L_{2}, \ldots, L_{m}$ are symmetric real entried matrices. Also we frequently abbreviate positive (resp. semi) definite to PD ( resp. PSD). A monic pencil is one with $L_{0}=I$. We call such a representation of a set $\mathcal{C}$ a Linear Matrix Inequality (LMI) representation of $\mathcal{C}$.

The question is formally stated by Pablo Parrilo and Berndt Sturmfels in [PSprep] for $m=2$, and we resolve the $m=2$ situation in this paper. At the end of the paper we speculate about generalizations and other possible representations (Section 6).

In this paper a polynomial always stands for a polynomial with real coefficients. Since the paper might be of interest to several audiences: operator theory, real semi-algebraic geometry, systems engineering and possibly partial differential equations, it is written less tersely than is common. Our result here was announced in a survey talk at the conference Mathematical Theory of Networks and Systems in 2002, see [Hprep].

1.1. Motivation. A technique which has had remarkable success in many types of optimization problems is to convert them to Linear Matrix Inequalities. These can be solved using semidefinite programming algorithms provided the number of variables $x_{j}$ is not extremely large. The technique was introduced first in the area of control in [BEFB94] and related papers and has spread through many part of that subject, c.f. [ESLSL00]. For a collection of applications in many other areas see the thesis [P00] and PSprep.

In the many applications which LMIs have found there is no systematic way to produce LMIs for general classes of problems. Each area has a few special tricks which convert "lucky problems" to LMIs. Before there is any hope of producing LMIs systematically one must have a good idea of which types of constraint sets convert to LMIs and which do not. That is what this paper addresses.

After the preliminary version of this paper was circulated as a preprint, Adrian Lewis, Pablo Parillo, and M. V. Ramana informed us that our main result was used in their solution, in the positive, of a 1958 conjecture by Peter Lax on hyperbolic polynomials arising from the study of linear hyperbolic partial differential equations; see LPRprep. 


\section{Solution}

In this section we give an algebraic statement of the solution to the Parrilo-Sturmfels problem, see Theorem [2.2. In Section 3] we give an equivalent geometric statement of the solution, see Theorem 3.1. In Section [5 we give an equivalent topological statement, see Corollary 5.1 and Theorem 5.2, which while intuitively appealing requires projective spaces to state in full generality. The solution depends on results of Victor Vinnikov V93. (see also Dubrovin [Dub83]) and variations on it.

2.1. A key class of polynomials. We shall soon see that it is a special class of polynomials we call real zero polynomials which bound sets amenable to LMI representation. So now we define a real zero polynomial ( RZ polynomial) to be a polynomial in $m$ variables satisfying for each $x \in \mathbb{R}^{m}$,

$$
p(\mu x)=0 \quad \text { implies } \quad \mu \quad \text { is real }
$$

A few properties are:

\section{RZ Properties}

(1) The product $p_{1} p_{2} \cdots p_{k}$ of $R Z$ polynomials $p_{1}, \ldots, p_{k}$ is a $R Z$ polynomial.

(2) If a $R Z$ polynomial $p$ factors as $p=p_{1} p_{2} \cdots p_{k}$, then all factors $p_{1}, p_{2}, \ldots, p_{k}$ are $R Z$ polynomials.

(3) The determinant

$$
\check{p}(x):=\operatorname{det}\left[L_{0}+L_{1} x_{1}+\cdots+L_{m} x_{m}\right]
$$

of a pencil $L$ with a PD matrix $L_{0}$, is an $R Z$ polynomial.

(4) The product of $\check{p}_{j}$ defined as determinants of pencils $L^{j}$ on $\mathbb{R}^{N_{j}}$, to wit

$$
\check{p}_{j}(x):=\operatorname{det}\left[L_{0}^{j}+L_{1}^{j} x_{1}+\cdots+L_{m}^{j} x_{m}\right]
$$

is also the determinant of a pencil $L$, namely, of the direct sum $L_{i}:=L_{i}^{1} \oplus \cdots \oplus L_{i}^{k}$ on a single $\mathbb{R}^{N}$ with $N=N_{1}+\cdots+N_{k}$. We have

$$
\begin{aligned}
\operatorname{det} L & =\left(\operatorname{det} L^{1}\right)\left(\operatorname{det} L^{2}\right) \ldots\left(\operatorname{det} L^{k}\right) \\
& =\check{p}_{1} \check{p}_{2} \ldots \check{p}_{k} .
\end{aligned}
$$

(5) See Section 3 and Section 5 for a characterization of $R Z$ polynomials in terms of geometric and topological properties of their zero sets. The topological characterization and related topological properties of $R Z$ polynomials, see Section 5.3, are essentially based on connectivity results of Nuij Nuij68. 


\section{Proofs:}

1. 2. The proofs of the first two properties about products and factors follows from the fact that the product $w=w_{1} \cdots w_{k}$ of a set of numbers is zero if and only if $w$ is zero.

3. For monic pencils

$$
\check{p}\left(\frac{x}{\lambda}\right)=\frac{1}{\lambda^{N}} \operatorname{det}\left[\lambda I+L_{1} x_{1}+\cdots+L_{m} x_{m}\right]
$$

and all of the eigenvalues of the symmetric matrix $L_{1} x_{1}+\cdots+L_{m} x_{m}$ are real, so we have that $\check{p}$ is $R Z$. Replacing the monic condition by $L_{0}$ is $\mathrm{PD}$ is obvious. A partial extension to the case when $L_{0}$ is merely PSD is provided by Lemma 2.3 below.

4. The proof is in the statement.

We start the presentation of our main result with a key definition.

2.2. Algebraic Interiors and their Degree. A closed set $\mathcal{C}$ in $\mathbb{R}^{m}$ is an Algebraic Interior, if there is a polynomial $p$ in $m$ variables such that $\mathcal{C}$ equals the closure of a connected component of

$$
\left\{x \in \mathbb{R}^{m}: p(x)>0\right\} .
$$

In other words, there is a polynomial $p$ in $m$ variables which vanishes on the boundary of $\mathcal{C}$ and such that $\{x \in \mathcal{C}: p(x)>0\}$ is connected with closure equal to $\mathcal{C}$. (Notice that in general $p$ may vanish also at some points in the interior of $\mathcal{C}$; for example, look at $p\left(x_{1}, x_{2}\right)=x_{2}^{2}-x_{1}^{2}\left(x_{1}-1\right)$.) We denote the closure of the connected component of $x^{0}$ in $\left\{x \in \mathbb{R}^{m}: p(x)>0\right\}$ by $\mathcal{C}_{p}\left(x^{0}\right)$. We often assume for normalization that 0 is in the interior of $\mathcal{C}$, and set $\mathcal{C}_{p}=\mathcal{C}_{p}(0)$.

Lemma 2.1. A polynomial $p$ of the lowest degree for which $\mathcal{C}=\mathcal{C}_{p}\left(x^{0}\right)$ is unique (up to a multiplication by a positive constant), and any other polynomial $q$ such that $\mathcal{C}=\mathcal{C}_{q}\left(x^{0}\right)$ is given by $q=p h$ where $h$ is an arbitrary polynomial which is strictly positive on a dense connected subset of $\mathcal{C}$.

Proof. We shall be using some properties of algebraic and semi-algebraic sets in $\mathbb{R}^{m}$, so many readers may want to skip over it and go to our main results which are much more widely understandable; our reference is BCR98. We notice first that $\mathcal{C}$ is a semialgebraic set (since it is the closure of a connected component of a semi-algebraic set, see BCR98, Proposition 2.2.2 and Theorem 2.4.5]). Therefore the interior int $\mathcal{C}$ of $\mathcal{C}$ is also semi-algebraic, and so is the boundary $\partial \mathcal{C}=\mathcal{C} \backslash \operatorname{int} \mathcal{C}$. Notice also that $\mathcal{C}$ equals the closure of its interior.

We claim next that for each $x \in \partial \mathcal{C}$, the local dimension $\operatorname{dim} \partial \mathcal{C}_{x}$ equals $m-1$. On the one hand, we have

$$
\operatorname{dim} \partial \mathcal{C}_{x} \leq \operatorname{dim} \partial \mathcal{C}<\operatorname{dim} \operatorname{int} \mathcal{C}=m
$$


here we have used BCR98, Proposition 2.8.13 and Proposition 2.8.4], and the fact that $\partial \mathcal{C}=\operatorname{clos} \operatorname{int} \mathcal{C} \backslash \operatorname{int} \mathcal{C}$, since $\mathcal{C}$ equals the closure of its interior. On the other hand, let $B$ be an open ball in $\mathbb{R}^{m}$ around $x$; then

$$
B \cap \partial \mathcal{C}=B \backslash\left[\left(B \cap\left(\mathbb{R}^{m} \backslash \mathcal{C}\right)\right) \cup(B \cap \operatorname{int} \mathcal{C})\right] .
$$

Since $\mathcal{C}$ equals the closure of its interior, every point of $\partial \mathcal{C}$ is an accumulation point of both $\mathbb{R}^{m} \backslash \mathcal{C}$ and int $\mathcal{C}$; therefore $B \cap\left(\mathbb{R}^{m} \backslash \mathcal{C}\right)$ and $B \cap \operatorname{int} \mathcal{C}$ are disjoint open nonempty semialgebraic subsets of $B$. Using [BCR98, Lemma 4.5.2] we conclude that $\operatorname{dim} B \cap \partial \mathcal{C} \geq m-1$, hence $\operatorname{dim} \partial \mathcal{C}_{x} \geq m-1$.

Let now $V$ be the Zariski closure of $\partial \mathcal{C}$, and let $V=V_{1} \cup \cdots \cup V_{k}$ be the decomposition of $V$ into irreducible components. We claim that $\operatorname{dim} V_{i}=m-1$ for each $i$. Assume by contradiction that $V_{1}, \ldots, V_{l}$ have dimension $m-1$ while $V_{l+1}, \ldots, V_{k}$ have smaller dimension. Then there exists $x \in \partial \mathcal{C}$ such that $x \notin V_{1}, \ldots, V_{l}$, and consequently there exists an open ball $B$ in $\mathbb{R}^{m}$ around $x$ such that

$$
B \cap \partial \mathcal{C}=\left(B \cap \partial \mathcal{C} \cap V_{l+1}\right) \cup \cdots \cup\left(B \cap \partial \mathcal{C} \cap V_{k}\right) .
$$

By assumption each set in the union on the right hand side has dimension smaller than $m-1$, hence it follows (by BCR98, Proposition 2.8.5, I]) that $\operatorname{dim} B \cap \partial \mathcal{C}<m-1$, a contradiction with $\operatorname{dim} \partial \mathcal{C}_{x}=m-1$.

Suppose now that $p$ is a polynomial of the lowest degree with $\mathcal{C}=\mathcal{C}_{p}\left(x^{0}\right)$. Lowest degree implies that $p$ can have no multiple irreducible factors, i.e., $p=p_{1} \cdots p_{s}$, where $p_{1}, \ldots, p_{s}$ are distinct irreducible polynomials; we may assume without loss of generality that every $p_{i}$ is non-negative on $\mathcal{C}$. Since $p$ vanishes on $\partial \mathcal{C}$ it also vanishes on $V=$ $V_{1} \cup \cdots \cup V_{k}$. We claim that for every $V_{i}$ there exists a $p_{j}$ so that $p_{j}$ vanishes on $V_{i}$ : otherwise $\mathcal{Z}\left(p_{j}\right) \cap V_{i}$ is a proper algebraic subset of $V_{i}$ for every $j=1, \ldots, s$, therefore (since $V_{i}$ is irreducible) $\operatorname{dim} \mathcal{Z}\left(p_{j}\right) \cap V_{i}<\operatorname{dim} V_{i}$ for every $j$ and thus also $\operatorname{dim} \mathcal{Z}(p) \cap V_{i}<\operatorname{dim} V_{i}$, a contradiction since $p$ vanishes on $V_{i}$. If $p_{j}$ vanishes on $V_{i}$, it follows ( since $p_{j}$ is irreducible and $\left.\operatorname{dim} V_{i}=m-1\right)$ that $\mathcal{Z}\left(p_{j}\right)=V_{i}$. The fact that $p$ is a polynomial of the lowest degree with $\mathcal{C}=\mathcal{C}_{p}\left(x^{0}\right)$ implies now (after possibly renumbering the irreducible factors of $p$ ) that $p=p_{1} \cdots p_{k}$ where $\mathcal{Z}\left(p_{i}\right)=V_{i}$ for every $i$. Since $\operatorname{dim} V_{i}=m-1$ it follows from the real Nullstellensatz for principal ideals [BCR98, Theorem 4.5.1] that the irreducible polynomials $p_{i}$ are uniquely determined (up to a multiplication by a positive constant), hence so is their product $p$. This proves the uniqueness of $p$.

The rest of the lemma now follows easily. If $\mathcal{C}=\mathcal{C}_{q}\left(x^{0}\right)$, then the polynomial $q$ vanishes on $\partial \mathcal{C}$ hence also on $V=V_{1} \cup \cdots \cup V_{k}$. Since $q$ vanishes on $\mathcal{Z}\left(p_{i}\right)=V_{i}$ and $\operatorname{dim} V_{i}=m-1$, the real Nullstellensatz for principal ideals implies that $q$ is divisible by $p_{i}$; this holds for every $i$ hence $q$ is divisible by $p=p_{1} \cdots p_{k}$, i.e., $q=p h$. It is obvious that $h$ must be strictly positive on a dense connected subset of $\mathcal{C}$. 
We refer to $p$ as a minimal defining polynomial for $\mathcal{C}$, and say that $\mathcal{C}$ is an Algebraic Interior of Degree $d$, where $d=\operatorname{deg} p$.

\subsection{Main Theorem on LMI Representations: Algebraic Version.}

Theorem 2.2. If a set $\mathcal{C}$ in $\mathbb{R}^{m}$, with 0 in the interior of $\mathcal{C}$, has an $L M I$ representation, then it is a convex algebraic interior and the minimal defining polynomial $p$ for $\mathcal{C}$ satisfies the $R Z$ condition (with $p(0)>0$ ).

Conversely, when $m=2$, if $p$ is an $R Z$ polynomial of degree $d$ and $p(0)>0$, then $\mathcal{C}_{p}$ has a monic LMI representation with $d \times d$ matrices.

The idea behind our organization of the proof is to do the informative elementary arguments first, then a geometric interpretation in Section 3 (readers less interested in proofs might go there next), and then some examples, and then in a seperate section do the converse side, which refers heavily to [V93.

2.3.1. Proof that $R Z$ is Necessary. We are given a pencil $L$ which represents $\mathcal{C}, \mathcal{C}=\mathcal{C}^{L}$; since $0 \in \mathcal{C}$ we have $L_{0}$ is PSD. Lemma 2.3 below reduces general pencils with $L_{0}$ a PSD matrix to monic pencils, so we assume that $L$ is a monic pencil. Define a polynomial $\check{p}$ by

$$
\check{p}(x):=\operatorname{det}\left[I+L_{1} x_{1}+\cdots+L_{m} x_{m}\right] .
$$

and note that $R Z$ Property 3 says that $\check{p}$ satisfies $R Z$. Now we show that $\mathcal{C}_{\check{p}}$ equals $\mathcal{C}^{L}$ (equals $\mathcal{C}$ ). If $x(t), t \in[0,1]$, is any continuous path in $\mathbb{R}^{m}$ with $x(0)=0$ and $\check{p}(x(t))>0$ for all $t$, then $L(x(t))$ is a continuous path in real symmetric matrices with $L(x(0))=I$ and $\operatorname{det} L(x(t))>0$ for all $t$, hence $L(x(1))$ is necessarily PD. It follows that the connected component of 0 in $\left\{x \in \mathbb{R}^{m}: \check{p}(x)>0\right\}$ is contained in $\mathcal{C}^{L}$ and therefore $\mathcal{C}_{\breve{p}}$ is contained in $\mathcal{C}^{L}$. Conversely, $x \in \mathcal{C}^{L}$ implies $I+\mu\left[L_{1} x_{1}+\cdots+L_{m} x_{n}\right]$ is $\mathrm{PD}$ for $0 \leq \mu<1$, so $\check{p}(\mu x)>0$ for $0 \leq \mu<1$ and therefore necessarily $x \in \mathcal{C}_{\check{p}}$.

We conclude that $\mathcal{C}=\mathcal{C}_{\breve{p}}$ is a convex algebraic interior. If $p$ is the minimal defining polynomial for $\mathcal{C}$, then $p$ is a factor of $\check{p}$. By $R Z$ Property 2 the polynomial $p$ is itself $R Z$.

Notice that the same argument as in the proof shows that for a monic pencil $L$ the interior of $\mathcal{C}=\mathcal{C}^{L}$ is exactly $\left\{x: I+x_{1} L_{1}+\cdots x_{m} L_{m}\right.$ is $\left.\mathrm{PD}\right\}$, and therefore the polynomial $\check{p}$ - and thus also the minimal defining polynomial $p$ - is stricly positive on the interior of $\mathcal{C}$. 
2.3.2. Reduction to Monic Pencils. We reduce the pencil representation problem to one involving monic pencils as promised.

Lemma 2.3. If $\mathcal{C}$ contains 0 in its interior and if there is a pencil $L$ such that

$$
\mathcal{C}=\mathcal{C}^{L}
$$

then there is a monic pencil $\widehat{L}$ such that

$$
\mathcal{C}=\mathcal{C}^{\widehat{L}}
$$

Proof. Given $\mathcal{C}$ and $L$, since $0 \in \mathcal{C}$ we have $0 \in \mathcal{C}^{L}$, or equivalently, $L_{0}$ is PSD. We need, however, to take $L_{0}$ invertible. Since 0 is in the interior of $\mathcal{C} \subset \mathbb{R}^{m}$, it is also in the interior of $C^{L}$, so we have

$$
L_{0} \geq \pm \varepsilon_{1} L_{1}, \ldots, L_{0} \geq \pm \varepsilon_{m} L_{m}
$$

for small enough $\varepsilon_{1}$ through $\varepsilon_{m}$. Set $Y=$ Range $L_{0} \subset \mathbb{R}^{N}$, and set

$$
\widetilde{L}_{j}:=\left.L_{j}\right|_{Y} \quad j=0,1, \ldots, m .
$$

Clearly $\widetilde{L}_{0}: Y \rightarrow Y$ is invertible and indeed PD. We next show that Range $L_{0}$ contains Range $L_{1}$. If $x \perp$ Range $L_{0}$, i.e., $L_{0} x=0$, then $0=x^{T} L_{0} x \geq \pm \varepsilon_{1} x^{T} L_{1} x$ and hence $x^{T} L_{1} x=0$. Since $L_{0}+\varepsilon_{1} L_{1} \geq 0$ and $x^{T}\left(L_{0}+\varepsilon_{1} L_{1}\right) x=0$ it follows that $\left(L_{0}+\varepsilon_{1} L_{1}\right) x=0$, and since $L_{0} x=0$, we finally conclude that $L_{1} x=0$, i.e., $x \perp$ Range $L_{1}$.

Likewise, Range $L_{0}$ contains the ranges of $L_{2}, \cdots, L_{m}$. Consequently, $\widetilde{L}_{j}: Y \rightarrow Y$ are all symmetric and $\mathcal{C}^{L}=\mathcal{C}^{\widetilde{L}}$.

To build $\widehat{L}$, factor $\widetilde{L}_{0}=B^{T} B$ with $B$ invertible and set

$$
\widehat{L}_{j}:=B^{-T} \widetilde{L}_{j} B^{-1} \quad j=0, \ldots, m .
$$

The resulting pencil $\widehat{L}$ is monic and $\mathcal{C}^{\widehat{L}}=\mathcal{C}^{\widetilde{L}}=\mathcal{C}^{L}$.

2.4. Shifted Real Zero Condition. Assuming that $0 \in \mathcal{C}$ is just a normalization. We can shift $\mathbb{R}^{m}$ by $x^{0}$ and obtain a shifted polynomial

$$
\tilde{p}(x):=p\left(x+x^{0}\right)
$$

with $\mathcal{C}_{p}\left(x^{0}\right)-x^{0}=\mathcal{C}_{\tilde{p}}(0)$. Then the $R Z$ condition on $\tilde{p}$ shifts back to the $R Z_{x^{0}}$ condition on $p$ at $x^{0}$. Namely, for each $x \in \mathbb{R}^{m}$

$$
p\left(\mu x+x^{0}\right)=0 \text { implies } \mu \text { is real. }
$$

An obvious consequence of our main theorem is: 
Corollary 2.4. If a set $\mathcal{C}$ in $\mathbb{R}^{m}$, with $x^{0}$ in the interior of $\mathcal{C}$, has an LMI representation, then it is a convex algebraic interior and the minimal defining polynomial $p$ for $\mathcal{C}$ satisfies the $R Z_{x^{0}}$ condition.

Conversely, when $m=2$, if $p$ is polynomial of degree $d$ satisfying $R Z_{x^{0}}$ and $p\left(x^{0}\right)>0$, then $\mathcal{C}_{p}\left(x^{0}\right)$ has an LMI representation with $d \times d$ matrices.

Remark. For $m=2$, "going through" LMI representations, i.e., invoking the second assertion of Corollary 2.4 followed by the first assertion, we conclude that

If the polynomial $p$ satisfies $R Z_{\tilde{x}^{0}}$ for some point $\tilde{x}^{0}$ and $p\left(\tilde{x}^{0}\right)>0$, then $p$ satisfies $R Z_{x^{0}}$ for every interior point $x^{0}$ of $\mathcal{C}_{p}\left(\tilde{x}^{0}\right)$.

This statement holds in any dimension $m$ and can be proved, without any reference to LMI representations, using the alternative topological characterization of $R Z$ polynomials. See Section 5.3 .

Proof of Corollary 2.4. Assume that a set $\mathcal{C}$ in $\mathbb{R}^{m}$, with $x^{0}$ in the interior of $\mathcal{C}$, has a pencil representation $L, \mathcal{C}=\mathcal{C}^{L}$. Shift $L$ by $x^{0}$ to obtain a pencil $\widetilde{L}$, that is,

$$
\widetilde{L}_{0}+\widetilde{L}_{1} x_{1}+\cdots+\widetilde{L}_{m} x_{m}=L_{0}+L_{1}\left(x_{1}+x_{1}^{0}\right)+\cdots+L_{m}\left(x_{m}+x_{m}^{0}\right) .
$$

Then $\mathcal{C}^{L}-x^{0}=\mathcal{C}^{\widetilde{L}}$, i.e., $\widetilde{L}$ is a pencil representation of the set $\widetilde{\mathcal{C}}=\mathcal{C}-x^{0}$, with 0 in the interior of $\widetilde{\mathcal{C}}$. By Lemma 2.3 we may assume that $\widetilde{L}$ is a monic pencil. The determinant $\check{\tilde{p}}$ of $\widetilde{L}$ satisfies the $R Z_{0}$ property. Thus the minimal defining polynomial $\tilde{p}$ of $\widetilde{\mathcal{C}}$, which is the shift of the minimal defining polynomial $p$ of $\mathcal{C}$ and which is contained in the $R Z_{0}$ polynomial $\check{\tilde{p}}$ as a factor, satisfies $R Z_{0}$. Shift 0 back to $x^{0}$ to get that $p$ satisfies $R Z_{x^{0}}$.

The proof of the second assertion, for $m=2$, proceeds analogously.

Notice a little more general than saying that the shifted pencil $\widetilde{L}$ is monic, is saying that $\widetilde{L}_{0}$ is PD. This is equivalent to saying that $L_{0}+x_{1}^{0} L_{1}+\cdots+x_{m}^{0} L_{m}$ is $\mathrm{PD}$, and then, by the remark at the end of Section 2.3.1, the interior of $\mathcal{C}=\mathcal{C}^{L}$ is exactly $\{x$ : $L_{0}+x_{1} L_{1}+\cdots x_{m} L_{m}$ is $\left.\mathrm{PD}\right\}$.

\section{Geometrical Viewpoint and Examples of Sets with no LMi REPRESENTATION}

In this section we give a geometric characterization of sets $\mathcal{C}$ with an LMI representation, which is equivalent to Theorem 2.2 . 
3.1. Rigid Convexity. An algebraic interior $\mathcal{C}$ of degree $d$ in $\mathbb{R}^{m}$ with minimal defining polynomial $p$ will be called rigidly convex provided for every point $x^{0}$ in the interior of $\mathcal{C}$ and a generic line $\ell$ through $x^{0}, \ell$ intersects the (affine) real algebraic hypersurface $p(x)=0$ in exactly $d$ points. 1 We soon show that rigid convexity of $\mathcal{C}_{p}$ is the same as $p$ having the (shifted) $R Z$ Property and as a consequence we obtain the following geometric version of our main theorem.

Theorem 3.1. If $\mathcal{C}$ is a closed convex set in $\mathbb{R}^{m}$ with an LMI representation, then $\mathcal{C}$ is rigidly convex. When $m=2$, the converse is true, namely, a rigidly convex degree $d$ set has an LMI representation with symmetric matrices $L_{j} \in \mathbb{R}^{d \times d}$.

\section{Proof.}

The theorem follows from Theorem 2.2 (more precisely, its shifted version Corollary 2.4) provided we can show that an algebraic interior $\mathcal{C}$ with minimal defining polynomial $p$ is rigidly convex if and only if $p$ satisfies the shifted $R Z$ condition. We now set about to prove this equivalence.

Let $\ell:=\left\{x \in \mathbb{R}^{m}: x=\mu v+x^{0}, \mu \in \mathbb{R}\right\}, v \in \mathbb{R}^{m}$, be a parameterization of a line through $x^{0}$ in the interior of $\mathcal{C}$. The points of intersection of the real algebraic hypersurface $p(x)=0$ with the line $\ell$ are parameterized by exactly those $\mu \in \mathbb{R}$ at which

$$
f(\mu):=p\left(\mu v+x^{0}\right)=0,
$$

and rigid convexity of $\mathcal{C}_{p}$ says that (for a generic $\ell$ ) there are exactly $d$ such distinct $\mu$. However, the degree of $p$ is $d$, so for a generic direction $v \in \mathbb{R}^{m}$, the degree of the polynomial $f$ equals $d$ (the exceptional directions are given by $p_{d}(v)=0$, where $p_{d}$ is the sum of degree $d$ terms in $p$ ). Thus these $f$, by the Fundamental Theorem of Algebra, have exactly $d$ zeroes $\mu \in \mathbb{C}$, counting multiplicities. Furthermore, for a generic direction $v$, all the zeroes are simple: multiple zeroes correspond to common zeroes of $f$ and $f^{\prime}=\sum_{i=1}^{m} v_{i} \frac{\partial p}{\partial x_{i}}$, that is to lines $\ell$ which pass through a point of intersection of algebraic hypersurfaces $p(x)=0$ and $\sum_{i=1}^{m} v_{i} \frac{\partial p}{\partial x_{i}}(x)=0$; but since $p$ is irreducible and $\operatorname{deg} \sum_{i=1}^{m} v_{i} \frac{\partial p}{\partial x_{i}}<\operatorname{deg} p$, this intersection is a proper algebraic subvariety of $p(x)=0$ hence of dimension at most $m-2$. (This argument applies to both real and complex zeroes, by considering real algebraic hypersurfaces and their complexifications, respectively.) Thus rigid convexity says precisely that all zeroes of $f$ are at real $\mu$, for $f$ arising from a generic $v$.

\footnotetext{
1 "Generic" means that the exceptional lines are contained in a proper algebraic subvariety (in $\left.\mathbb{P}^{m-1}(\mathbb{R})\right)$; when $m=2$ this means simply all but a finite number of lines. One can replace here "generic line" by "every line" if one takes multiplicities into account when counting the number of intersections, and also counts the intersections at infinity, i.e., replaces the affine real algebraic hypersurface $p\left(x_{1}, \ldots, x_{m}\right)=0$ by the projective real algebraic hypersurface $X_{0}^{d} p\left(X_{1} / X_{0}, \ldots, X_{m} / X_{0}\right)=0$.
} 
To obtain that this is equivalent to the $R Z_{x^{0}}$ Property for $p$ we give an elementary continuity argument which implies that all $f$ have only real zeroes. Pick an exceptional direction $v$ (which might make $f$ have degree less than $d$ ): if $f_{n}$ is a sequence of polynomials of degree $d$ in one variable with real coefficients such that every $f_{n}$ has only real zeroes, and if the polynomials $f_{n}$ converge coefficientwise to a polynomial $f$ (of degree less than or equal to $d$ ), then $f$ has only real zeroes.

Notice that it follows from Theorem 3.1 that for $m=2$ a rigidly convex set is in fact convex, and (as in the Remark following Corollary 2.4), it is enough to require the defining property of rigid convexity to hold for lines through a single point of $\mathcal{C}$. Again, this holds for any dimension $m$ and can be proved directly using the alternative topological characterization of $R Z$ polynomials - see Section 5.3. We also indicate there a strong stability property of rigidly convex algebraic interiors, and speculate about this stability being characteristic of rigid convexity.

\subsection{Examples.}

\subsubsection{Example 1. The polynomial}

$$
p\left(x_{1}, x_{2}\right)=x_{1}^{3}-3 x_{2}^{2} x_{1}-\left(x_{1}^{2}+x_{2}^{2}\right)^{2}
$$

has zero set shown in Figure 1.

The complement to $p=0$ in $\mathbb{R}^{2}$ consists of 4 components, 3 bounded convex components where $p>0$ and an unbounded component where $p<0$. Let us analyze one of the bounded components, say the one in the right half plane, $\mathcal{C}$ is the closure of

$$
\left\{x: p(x)>0, x_{1}>0\right\} .
$$

Does $\mathcal{C}$ have an LMI representation? To check this: fix a point $O$ inside $\mathcal{C}$, e.g. $O=(.7,0)$.

By Theorem 3.1 almost every line $l$ thru $(0,0.7)$ ( as in Figure 1) must intersect $p=0$ in 4 real points or the $R Z_{O}$ condition is violated. We can see from the picture in $\mathbb{R}^{2}$ that there is a continuum of real lines $\ell$ through $(0,0.7)$ intersecting $p=0$ in exactly two real points. Thus by Theorem 3.1 the set $\mathcal{C}$ does not have an LMI representation. (Since $p$ is irreducible it is the minimum defining polynomial for $\mathcal{C}$.)

\subsubsection{Example 2.}

$$
p\left(x_{1}, x_{2}\right)=1-x_{1}^{4}-x_{2}^{4}
$$

Clearly, $\mathcal{C}_{p}:=\{x: p(x) \geq 0\}$ has degree 4 but all lines in $\mathbb{R}^{2}$ through it intersect the set $p=0$ in exactly two places. Thus $\mathcal{C}_{p}$ is not rigidly convex. 


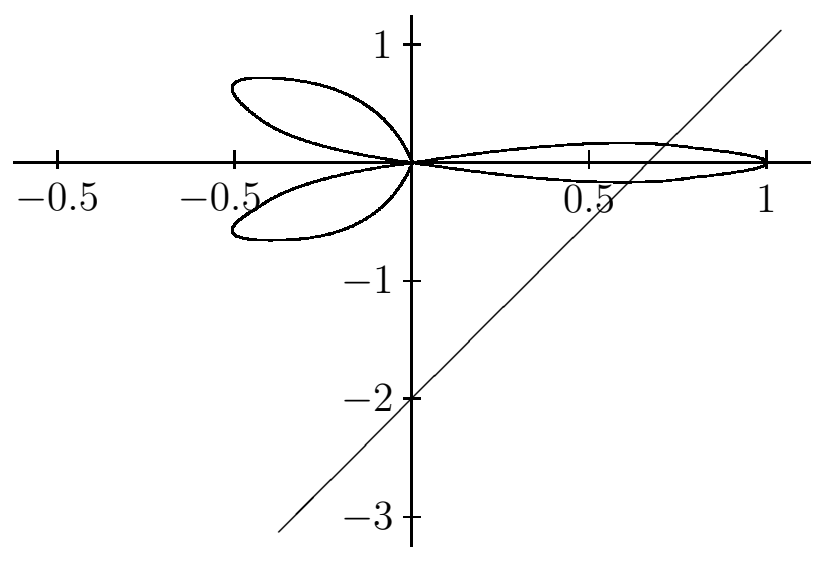

Figure 1. $p=x_{1}^{3}-3 x_{2}^{2} x_{1}-\left(x_{1}^{2}+x_{2}^{2}\right)^{2}$ with a line through $O=(.7,0)$ hitting $Z_{p}$ in only 2 points.

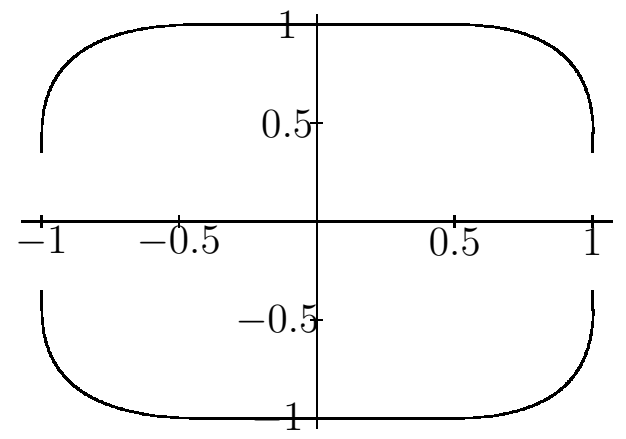

Figure 2. $p\left(x_{1}, x_{2}\right)=1-x_{1}^{4}-x_{2}^{4}$

\section{Proof of the CONVERSe Side of TheOrem 2.2}

A proof is an adaptation of the results of V93. Theorem 6.1 of V93 (see also Dub83) says that any polynomial $p$ on $\mathbb{R}^{2}$ satisfying $(R Z)$ (with $p(0)>0$ ) admits a representation (II) with $L_{0}, L_{1}$ and $L_{2}$ complex hermitian matrices on $\mathbb{C}^{d}, d=\operatorname{deg} p$, where $L_{0}$ is $\mathrm{PD}$. The possibility to take $L_{0}, L_{1}$ and $L_{2}$ real symmetric follows easily from the arguments given in the last 4 paragraphs of the Introduction (p. 456) of V93. It is assumed in V93] that $p$ is an irreducible polynomial, and the projective closure of $p\left(x_{1}, x_{2}\right)=0$ is a smooth projective plane curve; however the irreducibility assumption is irrelevant here since we may consider one irreducible factor of $p$ at a time (and then form a direct sum of the corresponding determinantal representations (1)), and the smoothness assumption can be easily removed by considering maximal determinantal representations as in [BV96, BV99]. 
This proof might lend itself to construction of the pencil $L$ via a construction of a basis for a linear system (or a Riemann Roch space) on a plane algebraic curve.

Since the proof above is actually an outline which leaves much for the reader to fill in, we present an alternative direct proof based on the results of [BV99, Section 5]. As before, we may assume without loss of generality that $p$ is an irreducible polynomial; we consider the complex affine algebraic curve $\left\{\left(x_{1}, x_{2}\right) \in \mathbb{C}^{2}: p\left(x_{1}, x_{2}\right)=0\right\}$, take its projective closure in the complex projective plane $\mathbb{P}^{2}(\mathbb{C})$, and let $M$ be the desingularizing Riemann surface. $M$ is a compact real Riemann surface, i.e., a compact Riemann surface equipped with an anti-holomorphic involution induced by $\left(x_{1}, x_{2}\right) \longrightarrow\left(\bar{x}_{1}, \bar{x}_{2}\right)$. The set $M_{\mathbb{R}}$ of real points of $M$ is by definition the set of fixed points of the anti-holomorphic involution, and $x_{1}$ and $x_{2}$ are real meromorphic functions on $M$, i.e., meromorphic functions taking real values at real points. Furthermore, it follows from the $R Z$ condition that the real meromorphic function $\frac{x_{2}}{x_{1}}$ on $M$ takes real values at real points only. It follows that $M$ is a compact real Riemann surface of dividing type, i.e., $M \backslash M_{\mathbb{R}}$ consists of two connected components. We denote by $M_{+}$and $M_{-}$the components where $\Im \frac{x_{2}}{x_{1}}>0$ and $\Im \frac{x_{2}}{x_{1}}<0$, respectively. We orient $M_{\mathbb{R}}$ so that $M_{\mathbb{R}}=\partial M_{+}$; then the real meromorphic differential $-d \frac{x_{1}}{x_{2}}=\frac{x_{1} d x_{2}-x_{2} d x_{1}}{x_{2}^{2}}$ is everywhere nonnegative on $M_{\mathbb{R}}$.

Since both the assumptions and the conclusions of the theorem are invariant under a linear change of coordinates $x_{1}, x_{2}$, we may assume without loss of generality that $p(0, \mu)=0$ has $d$ distinct real roots $c_{1}, \ldots, c_{d}$; they correspond to $d$ non-singular points $\left(0, c_{1}\right), \ldots,\left(0, c_{d}\right)$ on the affine algebraic curve $p\left(x_{1}, x_{2}\right)=0$, and we denote by $q_{1}, \ldots, q_{d}$ the corresponding points on $M$. We may now write down explicit formulae for the $d \times d$ matrices $L_{0}, L_{1}, L_{2}$ :

$$
\begin{aligned}
& L_{0}=I_{d}, \\
& {\left[L_{1}\right]_{i j}=\left\{\begin{array}{l}
i=j: \frac{\frac{d x_{2}}{d x_{1}}\left(q_{i}\right)}{c_{i}} \\
i \neq j:\left(\frac{1}{c_{i}}-\frac{1}{c_{j}}\right) \frac{\theta\left[\begin{array}{l}
a \\
b
\end{array}\right]\left(\phi\left(q_{j}\right)-\phi\left(q_{i}\right)\right)}{\theta\left[\begin{array}{l}
a \\
b
\end{array}\right](0) E\left(q_{j}, q_{i}\right)} \frac{1}{\sqrt{d \frac{x_{1}}{x_{2}}\left(q_{i}\right) \sqrt{d \frac{x_{1}}{x_{2}}}\left(q_{j}\right)}}, \\
L_{2}=\operatorname{diag}\left(-\frac{1}{c_{1}}, \ldots,-\frac{1}{c_{d}}\right) .
\end{array}\right.}
\end{aligned}
$$


Here $\theta\left[\begin{array}{l}a \\ b\end{array}\right](\cdot)$ is the theta function with characteristics associated to the Jacobian variety of $M, \phi$ is the Abel-Jacobi map from $M$ into the Jacobian variety, and $E(\cdot, \cdot)$ is the prime form on $M$; for details see [V93, Section 2] or [BV99, Section 4]. Furthermore, we choose a symmetric homology basis on $M$ as in [V93, Section 2] with a resulting period matrix $\Omega$, and $b+\Omega a \in \mathbb{C}^{g}$ is a half period in the Jacobian with $a_{1}, \ldots, a_{g+1-k}=1$ and $b_{g+2-k}, \ldots, b_{g}=0$ ( $g$ is the genus of $M$ and $k$ is the number of connected components of $\left.M_{\mathbb{R}}\right)$.

The fact that $\operatorname{det}\left(L_{0}+x_{1} L_{1}+x_{2} L_{2}\right)=p\left(x_{1}, x_{2}\right)$ (up to a constant positive factor) is exactly [BV99, Theorem 5.1] (with a pair of meromorphic functions $\lambda_{1}=1 / x_{1}, \lambda_{2}=x_{2} / x_{1}$ on $M$ and $-x_{1} / x_{2}$ as a local parameter at the poles of $\lambda_{1}$ and $\lambda_{2}$ (zeroes of $\left.x_{1}\right)$ ). The fact that $L_{1}$ is a real symmetric matrix follows immediately from the standard properties of theta functions and $\left[\mathrm{V93}\right.$, Proposition 2.3]. Notice that $b+\Omega a \in T_{0}$ hence $\theta\left[\begin{array}{l}a \\ b\end{array}\right](0) \neq 0$, see [V3, Theorem 3.1 and Corollary 4.3].

\section{Topological Characterization of $R Z$ Polynomials}

This section gives an equivalent topological characterization of zero sets of $R Z$ polynomials. First we show that $R Z$ polynomials of degree $d$ for which the sets of real zeros are compact are exactly those whose zero sets consist of a nest of $d / 2$ "ovaloids" (having compact zero set implies $d$ even). A result of similar definitiveness holds when real zero sets are not compact, but it requires projective space to neatly account for the effects of the zero set passing thru infinity. Thus we describe the general case only after describing $p$ with compact real zero set, despite generating a little redundancy

Then we give some related properties of $R Z$ polynomials.

5.1. $R Z$ Polynomials whose Sets of Real Zeroes are Compact. We first define carefully what is meant by an "ovaloid" in $\mathbb{R}^{m}$, and later give an analogous definition in projective space, see for example Viro86. We call $W \subset \mathbb{R}^{m}$ an ovaloid in $\mathbb{R}^{m}$ if $W$ is isotopic in $\mathbb{R}^{m}$ to a sphere $S \subset \mathbb{R}^{m}$, i.e., there is a homeomorphism $F$ of $\mathbb{R}^{m}$ with $F(S)=$ $W$, and furthermore $F$ is homotopic to the identity, i.e., there is a homeomorphism $H$ of $[0,1] \times \mathbb{R}^{m}$ such that $H_{t}=\left.H\right|_{\{t\} \times \mathbb{R}^{m}}$ is a homeomorphism of $\mathbb{R}^{m}$ for every $t, H_{0}=\operatorname{Id}_{\mathbb{R}^{m}}$, and $H_{1}=F$. Notice that $\mathbb{R}^{m} \backslash S$ consists of two connected components only one of which is contractible, hence the same is true of $\mathbb{R}^{m} \backslash W$; we call the contractible component the interior of the ovaloid $W$, and the non-contractible component the exterior.

The following is a consequence of Theorem 5.2 below. 
Corollary 5.1. Let $p$ be a polynomial whose set $V$ of real zeros is compact (then the degree $d$ of $p$ must be even, $d=2 k$ ) and which defines a smooth (affine) real algebraic hypersurface, i.e., $p$ has no critical points on $V$. Then $p$ satisfies $R Z_{x^{0}}$ with $p\left(x^{0}\right) \neq 0$ if and only if $V$ is a disjoint union of $k$ ovaloids $W_{1}, \ldots, W_{k}$ in $\mathbb{R}^{m}$, with $W_{i}$ contained in the interior of $W_{i+1}, i=1, \ldots, k-1$, and $x^{0}$ lying in the interior of $W_{1}$. Furthermore, in this case every line $L$ through $x^{0}$ intersects $V$ in $d$ distinct points.

The corollary misses being as strong as possible and in fact being sharp because of the requirement that ovaloids be disjoint. Intuitively, if the real zero set of a RZ polynomial is a compact singular (nonsmooth) hypersurface, then it consists of nested ovaloids that touch at the singular points, as in the figure below. Notice that it follows from Property 11 in Section 5.3 below that any $R Z$ polynomial of degree $d=2 k$ with a compact real zero set is the limit of $R Z$ polynomials of degree $d$ with compact real zero sets and which define smooth real algebraic hypersurfaces (that is, the zero sets are disjoint unions of $k$ nested ovaloids).

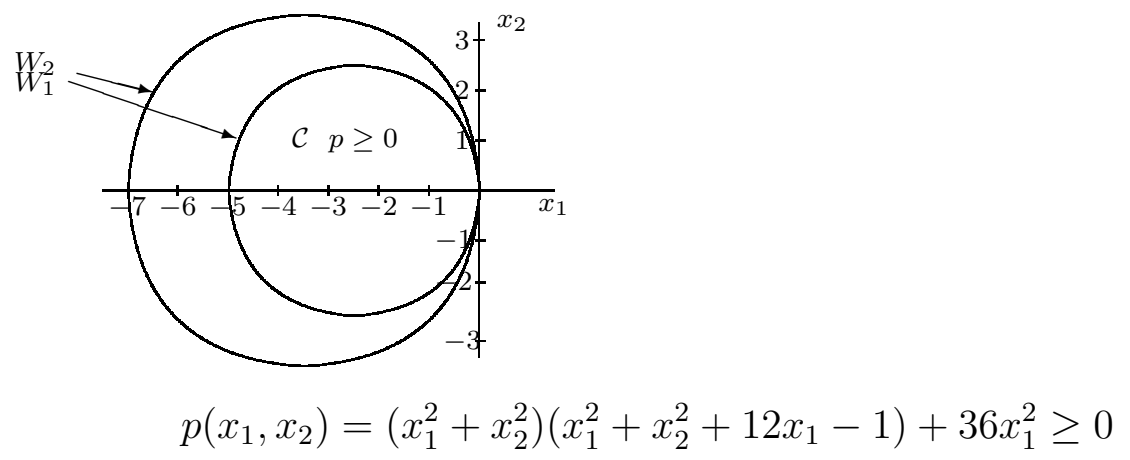

5.2. $R Z$ Polynomials with a Non-Compact Set of Real Zeroes. We shall need to consider the behavior of the polynomial at infinity, so we introduce the homogenization $P$ of $p$,

$$
P\left(X_{0}, X_{1}, \ldots, X_{m}\right)=X_{0}^{d} p\left(X_{1} / X_{0}, \ldots, X_{m} / X_{0}\right)
$$

where $d=\operatorname{deg} p$. Notice that $P$ is not divisible by $X_{0}$, and that conversely given any homogeneous polynomial $P$ of degree $d$ in $m+1$ variables which is not divisible by $X_{0}$ we may recover a polynomial $p$ of degree $d$ in $m$ variables so that $P$ is the homogenization of $p$ by

$$
p\left(x_{1}, \ldots, x_{m}\right)=P\left(1, x_{1}, \ldots, x_{m}\right) .
$$

We identify as usual the $m$ dimensional real projective space $\mathbb{P}^{m}(\mathbb{R})$ with the union of $\mathbb{R}^{m}$ and the hyperplane at infinity $X_{0}=0$, so that the affine coordinates $x$ and the projective coordinates $X$ are related by $x_{1}=X_{1} / X_{0}, \ldots, x_{m}=X_{m} / X_{0}$; the projective real algebraic hypersurface $P(X)=0$ in $\mathbb{P}^{m}(\mathbb{R})$ is called the projective closure of the affine real algebraic hypersurface $p(x)=0$ in $\mathbb{R}^{m}$. We shall say that $p$ defines a smooth projective real algebraic 
hypersurface if $P$ has no critical points on $P(X)=0$. We shall often abuse the notation and denote a point in $\mathbb{P}^{m}(\mathbb{R})$ and a $(m+1)$-tuple of its projective coordinates by the same letter.

We call $W \subset \mathbb{P}^{m}(\mathbb{R})$ an ovaloid if $W$ is isotopic in $\mathbb{P}^{m}(\mathbb{R})$ to a sphere $S \subset \mathbb{R}^{m} \subset$ $\mathbb{P}^{m}(\mathbb{R})$, i.e., there is a homeomorphism $F$ of $\mathbb{P}^{m}(\mathbb{R})$ with $F(S)=W$, and furthermore $F$ is homotopic to the identity, i.e., there is a homeomorphism $H$ of $[0,1] \times \mathbb{P}^{m}(\mathbb{R})$ such that $H_{t}=\left.H\right|_{\{t\} \times \mathbb{P}^{m}(\mathbb{R})}$ is a homeomorphism of $\mathbb{P}^{m}(\mathbb{R})$ for every $t, H_{0}=\operatorname{Id}_{\mathbb{P}^{m}(\mathbb{R})}$, and $H_{1}=F$. Notice that $\mathbb{P}^{m}(\mathbb{R}) \backslash S$ consists of two connected components only one of which is contractible, hence the same is true of $\mathbb{P}^{m}(\mathbb{R}) \backslash W$; we call the contractible component the interior of the ovaloid $W$, and the non-contractible component the exterior.

We call $W \subset \mathbb{P}^{m}(\mathbb{R})$ a pseudo-hyperplane if $W$ is isotopic in $\mathbb{P}^{m}(\mathbb{R})$ to a (projective) hyperplane $H \subset \mathbb{P}^{m}(\mathbb{R})$.

Theorem 5.2. Let $p$ be a polynomial of degree $d$ in $m$ variables which defines a smooth projective real algebraic hypersurface $V$ in $\mathbb{P}^{m}(\mathbb{R})$. Then $p$ satisfies $R Z_{x^{0}}$ with $p\left(x^{0}\right) \neq 0$ if and only if

a. if $d=2 k$ is even, $V$ is a disjoint union of $k$ ovaloids $W_{1}, \ldots, W_{k}$, with $W_{i}$ contained in the interior of $W_{i+1}, i=1, \ldots, k-1$, and $x^{0}$ lying in the interior of $W_{1}$;

b. if $d=2 k+1$ is odd, $V$ is a disjoint union of $k$ ovaloids $W_{1}, \ldots, W_{k}$, with $W_{i}$ contained in the interior of $W_{i+1}, i=1, \ldots, k-1$, and $x^{0}$ lying in the interior of $W_{1}$, and a pseudo-hyperplane $W_{k+1}$ contained in the exterior of $W_{k}$.

Furthermore, in this case every (projective) line $L$ through $x^{0}$ intersects $V$ in $d$ distinct points.

The proof appears in Section 7.

5.3. Topological Properties of $R Z$ Polynomials. We list now some properties of $R Z$ polynomials, mostly of a topological nature, which are closely related to Theorem 5.2 .

\section{RZ Topological Properties}

(1) If two polynomials $p_{0}, p_{1}$ of degree $d$ in $m$ variables satisfy $R Z$ and $p_{0}(0), p_{1}(0)>0$, then there is a continuous path through $R Z$ polynomials of degree $d$ in $m$ variables from $p_{0}$ to $p_{1}$ with each polynomial $p$ in the path satisfying $p(0)>0$. Furthermore, we may assume that except for possibly the endpoints $p_{0}, p_{1}$, the polynomial $p$ defines a smooth projective real algebraic hypersurface.

(2) If the polynomial $p$ satisfies $R Z_{\tilde{x}^{0}}$ for some point $\tilde{x}^{0}$ and $p\left(\tilde{x}^{0}\right)>0$, then $p$ satisfies $R Z_{x^{0}}$ for every interior point $x^{0}$ of $\mathcal{C}_{p}\left(\tilde{x}^{0}\right)$.

(3) If $p$ is a $R Z$ polynomial with $p(0)>0$ then the algebraic interior $\mathcal{C}_{p}$ is convex (so a rigidly convex algebraic interior is in fact convex). 
(4) Suppose that there is a continuous path through polynomials of degree $d$ in $m$ variables from a polynomial $p_{0}$ to a polynomial $p_{1}$, such that each polynomial $p$ in the path defines a smooth projective real algebraic hypersurface and $p(0)>0$. Then if $p_{0}$ is a $R Z$ polynomial, so is $p_{1}$.

(5) Suppose that there is a continuous path through polynomials of degree $d$ in $m$ variables from a polynomial $p_{0}$ to a polynomial $p_{1}$, such that each polynomial $p$ in the path including $p_{0}$ and $p_{1}$ defines a smooth projective real algebraic hypersurface. Assume that $p_{0}$ satisfies $R Z_{x^{0}}$ for some point $x^{0}$ and $p\left(x^{0}\right)>0$; then $p_{1}$ satisfies $R Z_{x^{1}}$ for some point $x^{1}$ and $p\left(x^{1}\right)>0$.

The proof appears in Section 7.

\section{Generalizations}

We now conjecture structure which extends that actually proved in this paper.

6.1. $m>2$. It is a natural question to which extent the converse side of our main results Theorem 2.2 and Theorem 3.1 extends to dimension $m>2$. A simple count of parameters shows that given a polynomial $p\left(x_{1}, \ldots, x_{m}\right)$ in $m>2$ variables, in general there do not exist $d \times d$ matrices $L_{0}, \ldots, L_{m}$ such that $p(x)=\operatorname{det}\left(L_{0}+x_{1} L_{1}+\cdots+x_{m} L_{m}\right)$ (here the polynomial has complex coefficients and the matrices are likewise complex). Thus we cannot expect a rigidly convex degree $d$ set in $\mathbb{R}^{m}, m>2$, to admit an LMI representation with $d \times d$ symmetric matrices. However the count of parameters does not preclude the existence of a pencil representation with matrices of a size larger than the degree. In fact we conjecture that

A convex set $\mathcal{C}$ in $\mathbb{R}^{m}$ has an LMI representation if and only if it is rigidly convex.

6.2. LMI Lifts. Another practically important question (in the spirit of Nestrov and Nimmerovski [NN94]) is

Question Find a test which insures that a convex set $\mathcal{C}$ in $\mathbb{R}^{m}$ lifts to some LMI representable set $\tilde{\mathcal{C}}$ in a possibly bigger space $\mathbb{R}^{m+k}$. That is, find neccesary and sufficient properties on a given set $\mathcal{C}$ in $\mathbb{R}^{m}$ which insure that there exists an LMI representable set $\tilde{\mathcal{C}}$ in $\mathbb{R}^{m+k}$ whose projection onto $\mathbb{R}^{m}$ equals the set $\mathcal{C}$ ?

No apriori restriction on such a $\mathcal{C}$ is appearent, either from theoretical considerations or from numerical experiments (done informally by Pablo Parrilo). Of course, $\mathcal{C}$ has to be a semi-algebraic set with a connected interior, which is equal to the closure of its interior. It is not clear whether $\mathcal{C}$ should be an algebraic interior as we defined it. 
6.3. Convexity under Deformation is Ephemeral. Properties 4 and 5 in Section 5.3 imply that the convexity of a rigidly convex algebraic interior $\mathcal{C}$ is stable under deformation of the minimal defining polynomial $p$ as long as the projective real algebraic hypersurface defined by $p$ (the projective closure in $\mathbb{P}^{m}(\mathbb{R})$ of the Zariski closure of $\partial \mathcal{C}$ in $\mathbb{R}^{m}$ ) remains smooth. We conjecture that this property characterizes rigidly convex algebraic interiors; more precisely, we make the following conjecture:

Let $\mathcal{C}_{0}=\mathcal{C}_{p_{0}}$ be a convex algebraic interior of degree $d$ in $\mathbb{R}^{m}$ which is not rigidly convex and such that the polynomial $p_{0}$ defines a smooth projective real algebraic hypersurface. Then there exists a continuous path through polynomials of degree $d$ in $m$ variables from a polynomial $p_{0}$ to a polynomial $p_{1}$, such that each polynomial $p$ in the path defines a smooth projective real algebraic hypersurface and $p(0)>0$, and the algebraic interior $\mathcal{C}_{p_{1}}$ is not convex.

For more information about various connected components of the set of polynomials of degree $d$ in $m$ variables that define a smooth projective real algebraic hypersurface, see Viro86.

\section{Appendix: Proofs of Topological Properties}

We first establish Property 1 of Section 5.3, and use it to prove Theorem 5.2. Then we establish Properties 2 [5.

Proof of Property 1. This crucial arcwise connectivity property follows from the results of Nuij Nuij68 on hyperbolic polynomials. Recall that a homogeneous polynomial $P$ in $m+1$ variables is said to be hyperbolic with respect to $X^{0}=\left(X_{0}^{0}, X_{1}^{0}, \ldots, X_{m}^{0}\right) \in$ $\mathbb{R}^{m+1}$ if $P\left(X^{0}\right) \neq 0$ and for each $X \in \mathbb{R}^{m+1}$ all the zeroes $\lambda$ of $P\left(X+\lambda X^{0}\right)$ are real. It is called strictly hyperbolic if all the zeroes are furthermore simple for each $X \in \mathbb{R}^{m+1}$ (not a multiple of $\left.X^{0}\right)$, and it is called normalized if $P\left(X^{0}\right)=1$.

It is immediate that a polynomial $p$ in $m$ variables satisfies $R Z_{x^{0}}$ and $p\left(x^{0}\right) \neq 0$ for $x^{0}=\left(x_{1}^{0}, \ldots, x_{m}^{0}\right)$ if and only if its homogenization $P$ is hyperbolic with respect to $X^{0}=$ $\left(1, x_{1}^{0}, \ldots, x_{m}^{0}\right)$. Notice that if $P$ is strictly hyperbolic, then necessarily $p$ defines a smooth projective real algebraic hypersurface $V .{ }^{2}$ For (as in the proof of Theorem 3.1) the zeroes of $P\left(X+\lambda X^{0}\right)$ correspond to intersections of the line through $X$ and $X^{0}$ with $V$; if $X$ were a singular point of $V$, the intersection multiplicity at $X$ of any line through $X$ with $V$ would be greater than 1 and the corresponding zero of $P\left(X+\lambda X^{0}\right)$ could not be simple.

\footnotetext{
${ }^{2}$ It will follow from Theorem 5.2 that the converse is also true - if $p$ defines a smooth projective real algebraic hypersurface $V$, then $P$ is strictly hyperbolic.
} 
Property 1 now follows from the following results of Nuij68 (where the polynomials are of degree $d$ and are hyperbolic with respect to a fixed $X^{0}$ ): (a) the set of strictly hyperbolic homogeneous polynomials is open; (b) every hyperbolic homogeneous polynomial is the end point of an open continuous path of strictly hyperbolic homogeneous polynomials; (c) the set of normalized strictly hyperbolic homogeneous polynomials is arcwise connected. Notice that since $X^{0}=\left(1, x_{1}^{0}, \ldots, x_{m}^{0}\right)$, homogeneous polynomials which are divisible by $X_{0}$ form an affine subspace of codimension at least 2 in the affine space of homogeneous polynomials $P$ with $P\left(X^{0}\right)=1$; so the set of normalized strictly hyperbolic homogeneous polynomials which are not divisible by $X_{0}$ is still arcwise connected.

Proof of Theorem [5.2, first direction. Assume that $p_{0}$ is a $R Z$ polynomial of degree $d$ in $m$ variables with $p_{0}(0)>0$ which defines a smooth projective real algebraic hypersurface $V_{0}$ in $\mathbb{P}^{m}(\mathbb{R})$. Let

$$
p_{1}\left(x_{1}, \ldots, x_{m}\right)=\left(r_{1}^{2}-x_{1}^{2}-\cdots-x_{m}^{2}\right) \cdots\left(r_{k}^{2}-x_{1}^{2}-\cdots-x_{m}^{2}\right)
$$

if $d=2 k$ is even, and

$$
p_{1}\left(x_{1}, \ldots, x_{m}\right)=\left(r_{1}^{2}-x_{1}^{2}-\cdots-x_{m}^{2}\right) \cdots\left(r_{k}^{2}-x_{1}^{2}-\cdots-x_{m}^{2}\right)\left(r_{k+1}-x_{1}\right)
$$

if $d=2 k+1$ is odd, for some $0<r_{1}<\cdots<r_{k}<r_{k+1}$. By Property 11 there exists a continuous path $\left\{p_{t}\right\}_{0 \leq t \leq 1}$ from $p_{0}$ to $p_{1}$ through $R Z$ polynomials of degree $d$ in $m$ variables such that $p_{t}(0)>0$ and $p_{t}$ defines a smooth projective real algebraic hypersurface $V_{t}$ in $\mathbb{P}^{m}(\mathbb{R})$ for each $t$. It follows from the Thom Isotopy Theorem (see BCR98, Theorem 14.1.1] or AbRo67, Theorem 20.2]) that there exists a homeomorphism $H$ of $[0,1] \times \mathbb{P}^{m}(\mathbb{R})$ such that $H_{t}=\left.H\right|_{\{t\} \times \mathbb{P}^{m}(\mathbb{R})}$ is a homeomorphism of $\mathbb{P}^{m}(\mathbb{R})$ for every $t, H_{0}=\operatorname{Id}_{\mathbb{P}^{m}(\mathbb{R})}$, and $H_{t}\left(V_{0}\right)=V_{t}$. Therefore $V_{0}$ is isotopic to $V_{1}$, and $V_{1}$ by construction is a disjoint union of $k$ nested ovaloids if $d=2 k$ and a disjoint union of $k$ nested ovaloids and a pseudo-hyperplane if $d=2 k+1$, thus $V_{0}$ is also, as is required.

It remains only to show that 0 is contained in the interior of the innermost ovaloid. Let $x^{t}=H_{t}^{-1}(0)$; since $p_{t}(0)>0$ for all $t, 0 \notin V_{t}$ and hence $x^{t} \notin V_{0}$. Thus $x^{t}$ is a continuous path in $\mathbb{P}^{m}(\mathbb{R}) \backslash V_{0}$. Since 0 obviously belongs to the interior of the innermost sphere in $V_{1}$, it follows that $x^{1}$ belongs to the interior of the innermost ovaloid in $V_{0}$. Therefore so does $x^{0}=0$.

Proof of Theorem [5.2, second direction. Assume now conversely that $p$ is a polynomial of degree $d$ in $m$ variables that defines a smooth projective real algebraic hypersurface $V$ in $\mathbb{P}^{m}(\mathbb{R})$ which is a disjoint union of $k$ nested ovaloids $W_{1}, \ldots, W_{k}$ if $d=2 k$ and a disjoint union of $k$ nested ovaloids $W_{1}, \ldots, W_{k}$ and a pseudo-hyperplane $W_{k+1}$ if $d=2 k+1$. Assume that $X^{0}$ is in the interior of $W_{1}$, and let $L$ be a (projective) line in $\mathbb{P}^{m}(\mathbb{R})$ through $X^{0}$.

It is obvious that there exists a pseudo-hyperplane $W$ contained in the exterior of the outermost ovaloid $W_{k}$ (just take the image of a hyperplane contained in the exterior of 
a sphere $S$ in $\left.\mathbb{P}^{m}(\mathbb{R})\right)$. Since the $\mathbb{Z} / 2$ fundamental class of $W$ coincides with that of a hyperplane, it follows that the $\mathbb{Z} / 2$ intersection number of $L$ and $W$ equals 1 , hence $L \cap W \neq \emptyset$. Let $X \in L \cap W$; in case $d=2 k+1$ is odd we take $W=W_{k+1}$ and denote $X^{k+1}=X$.

The points $X^{0}$ and $X$ decompose the projective line $L$ into two closed intervals $L_{+}$and $L_{-}$. Each of $L_{ \pm}$is a continuous path between $X^{0}$ which lies in the interior of $W_{1}$ and $X$ which lies in the exterior of $W_{k}$; therefore $L_{ \pm}$intersects each of $W_{1}, \ldots, W_{k}$ in at least one point $X_{ \pm}^{1}, \ldots, X_{ \pm}^{k}$. Adding to these $X^{k+1}$ in case $d=2 k+1$ is odd, we obtain $d$ distinct points of intersection of $L$ with $V$; since the defining polynomial $p$ of $V$ has degree $d$, $L \cap V$ can contain at most $d$ points, so we conclude that $L \cap V$ contains exactly $d$ distinct points $X_{ \pm}^{1}, \ldots, X_{ \pm}^{k}$ and (in case $d=2 k+1$ is odd) $X^{k+1}$. This completes the proof. Notice that it follows that on $L_{ \pm}$we have the following picture: the interval $\left[X^{0}, X_{ \pm}^{1}\right)$ lies in the interior of $W_{1}$, the interval $\left(X_{ \pm}^{1}, X_{ \pm}^{2}\right)$ lies in the interior of $W_{2}$ intersection the exterior of $W_{1}, \ldots$, the interval $\left(X_{ \pm}^{k-1}, X_{ \pm}^{k}\right)$ lies in the interior of $W_{k}$ intersection the exterior of $W_{k-1}$, and the interval $\left(X_{ \pm}^{k}, X\right]$ lies in the exterior of $W_{k}$.

Proof of Property 2, By Property 1, we may assume, using approximation, that the polynomial $p$ defines a smooth projective real algebraic hypersurface. The conclusion now follows immediately from Theorem 5.2 .

Proof of Property 3. We may assume, again, that the polynomial $p$ defines a smooth projective real algebraic hypersurface $V$ in $\mathbb{P}^{m}(\mathbb{R})$. By Theorem 5.2, $V$ is a disjoint union of $k$ nested ovaloids $W_{1}, \ldots, W_{k}$ if $d=2 k$ and a disjoint union of $k$ nested ovaloids $W_{1}, \ldots, W_{k}$ and a pseudo-hyperplane $W_{k+1}$ if $d=2 k+1 ; X^{0}=(1,0, \ldots, 0)$ (we are using here projective coordinates) lies in the interior $\mathcal{I}$ of $W_{1}$.

It is obvious that $\mathcal{C}_{p}$ is the closure of the connected component of $X^{0}$ in $\mathcal{I} \backslash \mathcal{I} \cap H_{\infty}$ where $H_{\infty}=\left\{X_{0}=0\right\}$ is the hyperplane at infinity. We shall show that either $\mathcal{I} \cap H_{\infty}=\emptyset$ in which case $\mathcal{I} \subset \mathbb{R}^{m}$ is convex, or $\mathcal{I} \backslash \mathcal{I} \cap H_{\infty}$ consists of two connected convex components in $\mathbb{R}^{m}$.

Before proceeding, let us notice that there exists a hyperplane $H$ in $\mathbb{P}^{m}(\mathbb{R})$ such that $H \cap \mathcal{I}=\emptyset$. We may take $H$ to be the (projectivized) tangent hyperplane to $V$ at a point $Y^{0} \in W_{1}$. If $H$ contained a point $Y$ in $\mathcal{I}$, then by Theorem 5.2 the line through $Y$ and $Y^{0}$ would intersect $V$ in $d$ distinct points; but since this line is contained in $H$ it has intersection multiplicity at least 2 with $V$ at $Y^{0}$, so it cannot intersect $V$ in more than $d-1$ distinct points.

Let now $Y^{1}, Y^{2} \in \mathcal{I} \backslash \mathcal{I} \cap H_{\infty}$. Let $L$ be the projective line through $Y^{1}$ and $Y^{2}$ and let $X^{\infty}=L \cap H_{\infty}$. The two points $Y^{1}$ and $Y^{2}$ decompose the projective line $L$ into two closed intervals; the line segment between $Y^{1}$ and $Y^{2}$ in $\mathbb{R}^{m}$ is the interval that does not contain 
the point $X^{\infty}$. Notice that by the last sentence in the proof of Theorem 5.2, exactly one of the two intervals is contained in $\mathcal{I}$; let us denote it by $L_{\mathcal{I}}$.

Assume that $\mathcal{I} \cap H_{\infty}=\emptyset$. Then $X^{\infty} \notin \mathcal{I}$ and therefore $X^{\infty} \notin L_{\mathcal{I}}$. It follows that $L_{\mathcal{I}}$ is the line segment between $Y^{1}$ and $Y^{2}$ in $\mathbb{R}^{m}$, and $\mathcal{I} \subset \mathbb{R}^{m}$ is convex.

If $\mathcal{I} \cap H_{\infty} \neq \emptyset$, let $a_{0} X_{0}+a_{1} X_{1}+\cdots+a_{m} X_{m}=0$ be a hyperplane in $\mathbb{P}^{m}(\mathbb{R})$ that does not intersect $\mathcal{I}$. Let $\mathcal{I}_{+}$and $\mathcal{I}_{-}$be the nonempty open subsets of $\mathcal{I} \backslash \mathcal{I} \cap H_{\infty}$ where $X_{0} /\left(a_{0} X_{0}+a_{1} X_{1}+\cdots+a_{m} X_{m}\right)>0$ and $X_{0} /\left(a_{0} X_{0}+a_{1} X_{1}+\cdots+a_{m} X_{m}\right)<0$, respectively. We shall show that $\mathcal{I}_{+}$and $\mathcal{I}_{-}$are convex subsets of $\mathbb{R}^{m}$, thereby completing the proof. It is enough to show that if the line segment between $Y^{1}$ and $Y^{2}$ in $\mathbb{R}^{m}$ is not contained in $\mathcal{I}$ and say $Y^{1} \in \mathcal{I}_{+}$, then necessarily $Y^{2} \in \mathcal{I}_{-}$.

Assume then that the line segment between $Y^{1}$ and $Y^{2}$ in $\mathbb{R}^{m}$ is not contained in $\mathcal{I}$. This means that this line segment is not $L_{\mathcal{I}}$, so $X^{\infty} \in L_{\mathcal{I}}$. It is now immediate that the function $X_{0} /\left(a_{0} X_{0}+a_{1} X_{1}+\cdots+a_{m} X_{m}\right)$ on $L_{\mathcal{I}}$ changes its sign at $X^{\infty}$.

Proof of Property 4. This follows immediately from the facts that a limit of $R Z$ polynomials is a $R Z$ polynomial (see the last sentence in the proof of Theorem 3.1), that a $R Z$ polynomial $p, p(0)>0$, defines a smooth projective real algebraic hypersurface if and only if its homogenization $P$ is strictly hyperbolic (with respect to $X^{0}=(1,0, \ldots, 0)$ ), and that the set of strictly hyperbolic homogeneous polynomials is open Nuij68.

(We could also prove Property 4 by using Theorem 5.2 and an isotopy argument, like the proof of Property 5 below.)

Proof of Property 5. By Thom Isotopy Theorem, smooth projective real algebraic hypersurfaces $V_{0}$ and $V_{1}$ in $\mathbb{P}^{m}(\mathbb{R})$ defined by the polynomials $p_{0}$ and $p_{1}$ respectively are isotopic; the result now follows from Theorem 5.2.

\section{THANKS}

We are grateful to Bernd Sturmfels for suggesting this problem and to Pablo Parrilo for discussions bearing on possible extensions of these results.

Thanks for Helton's support are due to the NSF, DARPA, and the Ford Motor company. Thanks for Vinnikov's support are due to the Israel Science Foundation.

\section{REFERENCES}

[AbRo67] R. Abraham and J. Robbin, Transversal Mappings and Flows, Benjamin, New York, 1967.

[BV96] J. A. Ball and V. Vinnikov, Zero-pole interpolation for meromorphic matrix functions on an algebraic curve and transfer functions of 2D systems, Acta Appl. Math. 45 (1996), 239-316.

[BV99] _ Zero-pole interpolation for meromorphic matrix functions on a compact Riemann surface and a matrix Fay trisecant identity, Amer. J. Math. 121 (1999), 841-888. 
[BCR98] J. Bochnak, M. Coste, and M.-F. Roy, Real Algebraic Geometry, Springer-Verlag, BerlinHeidelberg, 1998.

[BEFB94] S. Boyd, L. El Ghaoui, E. Feron, and V. Balakrishna, Linear Matrix Inequalities In System and Control Theory, SIAM, 1994, pp. 193.

[Dub83] B. A. Dubrovin, Matrix finite zone operators, Contemporary Problems of Mathematics (Itogi Nauki i Techniki) 23, 33-78 (1983) (Russian).

[ESLSL00] El Ghaoui, Laurent and Niculescu, Siliviu-Lilian eds. Advances in Linear Matrix Inequality Methods in Control, Adv. Des. and Control, SIAM, 2000.

[Hprep] J. W. Helton, Manipulating Matrix Inequalities Autmatically, volume of Plenary Talks at the MTNS 2002, Springer Verlag, to appear

[LPRprep] A. S. Lewis, P. A. Parillo, and M. V. Ramana, The Lax conjecture is true, preprint.

[NN94] Yurii Nestrov and Arkadii Nimmerovski, Interior Point Polynomial Algorithms in Convex Programming, SIAM Studies 13, 1994, pp. 405.

[Nuij68] W. Nuij, A note on hyperbolic polynomials, Math. Scand. 23, 69-72 (1968).

[P00] P. Parrilo, Structured Semidefinite Programs and Semialgebraic Geometry Methods in Robustness and Optimization, PhD Thesis Cal Tech May 2000, available at http://www.cds.caltech.edu/ pablo

[PSprep] Pablo A. Parrilo and Bernd Sturmfels, Minimizing Polynomial Functions, preprint, pp. 16.

[V93] V. Vinnikov, Self-adjoint determinantal representions of real plane curves, Math. Ann. 296 (1993), 453-479.

[Viro86] O. Viro, Progress in the topology of real algebraic varieties over the last six years, Russian Math. Surveys 41, 55-82 (1986). 


\section{NOT FOR PUBLICATION}

\section{Contents}

1. The Problem of Representing Sets with LMI's 2

\begin{tabular}{ll} 
1.1. Motivation 2 & 2 \\
\hline
\end{tabular}

2. Solution 3

2.1. A kev class of polvnomials 3

2.2. Algebraic Interiors and their Degree 4

2.3. Main Theorem on LMI Representations: Algebraic Version 6

$\begin{array}{lll}\text { 2.4. Shifted Real Zero Condition } & 7\end{array}$

3. Geometrical Viewpoint and Examples of Sets with no LMI Representation 8

3.1. Rigid Convexity 9

$\begin{array}{ll}3.2 . \quad \text { Examples } & 10\end{array}$

4. Proof of the converse side of Theorem $2.2 \quad 11$

5. Topological Characterization of $R Z$ Polvnomials 13

$\begin{array}{lll}\text { 5.1. } R Z \text { Polynomials whose Sets of Real Zeroes are Compact } & 13\end{array}$

$\begin{array}{lll}\text { 5.2. } R Z \text { Polvnomials with a Non-Compact Set of Real Zeroes } & 14\end{array}$

5.3. Topological Properties of $R Z$ Polvnomials 15

$\begin{array}{ll}\text { 6. Generalizations } & 16\end{array}$

$\begin{array}{lr}6.1 . \quad m>2 & 16\end{array}$

$\begin{array}{ll}\text { 6.2. LMI Lifts } & 16\end{array}$

\begin{tabular}{lll}
\hline 6.3. Convexity under Deformation is Ephemeral & 17
\end{tabular}

\begin{tabular}{ll}
\hline 7. Appendix: Proofs of Topological Properties & 17
\end{tabular}

$\begin{array}{ll}8 . \text { Thanks } & 20\end{array}$

References 20 